\title{
Za

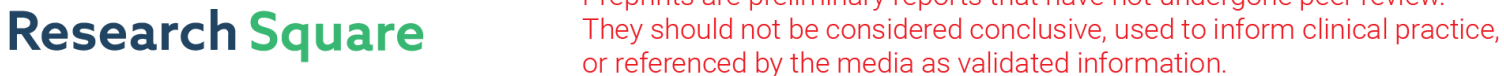

\section{IC100: A Novel Anti-Asc Monoclonal Antibody Improves Functional Outcomes In An Animal Model Of Multiple Sclerosis}

\section{Haritha L Desu}

University of Miami School of Medicine

Melanie Plastini

University of Miami School of Medicine

Placido Illiano

University of Miami School of Medicine

Helen M Bramlett

University of Miami School of Medicine

W. Dalton Dietrich

University of Miami School of Medicine

Juan Pablo de Rivero Vaccari

University of Miami School of Medicine

Roberta Brambilla

University of Miami School of Medicine

Robert W. Keane ( $\nabla$ rkeane@miami.edu )

University of Miami School of Medicine https://orcid.org/0000-0003-4068-9511

\section{Research}

Keywords: IC100, inflammasome, neuroinflammation, multiple sclerosis, experimental autoimmune encephalomyelitis, caspase-1, IL-1, ASC, Pycard

Posted Date: April 29th, 2020

DOl: https://doi.org/10.21203/rs.3.rs-17357/v2

License: (c) (i) This work is licensed under a Creative Commons Attribution 4.0 International License. Read Full License

Version of Record: A version of this preprint was published at Journal of Neuroinflammation on May 4th, 2020. See the published version at https://doi.org/10.1186/s12974-020-01826-0. 


\section{Abstract}

Background The inflammasome adaptor apoptosis-associated speck-like protein containing a CARD (ASC) is involved in immune signaling by bridging the interactions between inflammasome sensors and caspase-1. Strong experimental evidence has shown that $\mathrm{ASC}^{-/-}$mice are protected from disease progression in animal models of multiple sclerosis (MS), suggesting that targeting inflammasome activation via ASC inhibition may be a promising therapeutic strategy in MS. Thus, the goal of our study is to test the efficacy of IC100, a novel humanized antibody targeting ASC, in preventing and/or suppressing disease in the experimental autoimmune encephalomyelitis (EAE) model of MS.

Methods We employed the EAE model of MS where disease was induced by immunization of C57BL/6 mice with myelin oligodendrocyte glycoprotein peptide 35-55 $\left(\mathrm{MOG}_{35-55}\right)$. Mice were treated with vehicle or increasing doses of IC100 (10,30 and $45 \mathrm{mg} / \mathrm{kg})$ and clinical disease course was evaluated up to 35 days post EAE induction. Immune cell infiltration into the spinal cord and microglia responses were assessed.

Results We show that IC100 treatment reduced the severity of EAE when compared to vehicle-treated controls. At a dose of $30 \mathrm{mg} / \mathrm{kg}$, IC100 significantly reduced the number of CD $4^{+}$and $\mathrm{CD} 8^{+} \mathrm{T}$ cells and $\mathrm{CD} 11 \mathrm{~b}^{+} \mathrm{MHCII}^{+}$activated myeloid cells entering the spinal cord from the periphery, and reduced the number of total and activated microglia.

Conclusions These data indicate that IC100 suppresses the immune-inflammatory response that drives EAE development and progression, thereby identifying ASC as a promising target for the treatment of MS as well as other neurological diseases with a neuroinflammatory component.

\section{Background}

Multiple Sclerosis (MS) is an autoimmune demyelinating disease of the central nervous system (CNS) characterized by an inflammatory response sustained by innate and adaptive immune mechanisms dependent on lymphocyte (both $T$ and $B$ cells) and myeloid cell activation [1]. The direct immune attack on myelin producing oligodendroglia causes demyelination, leading to axonal and neuronal damage that correlates with long term disability [2]. Approximately $85 \%$ of MS patients present with a relapsingremitting phenotype (RRMS) and the majority of these evolve into a secondary-progressive (SPMS) disease course after 15 to 20 years. Approximately $10-15 \%$ of patients experience a primary progressive (PPMS) disease course with slow and continuous deterioration without definable relapses [3]. The understanding of MS pathomechanisms as well as the development of MS therapeutics have relied upon the use of the experimental autoimmune encephalomyelitis (EAE) model. In fact, many of the diseasemodifying drugs currently in clinical use have been developed after successful testing in EAE [4].

Inflammasomes are the central signaling platforms of the innate immune-inflammatory response. Upon activation by a diverse range of intracellular sensors of infection, cell damage or metabolic disturbances, 
the adaptor protein apoptosis speck-like staining protein containing a caspase recruitment domain (ASC), common to all inflammasomes, is recruited. Sensor oligomerization induces the polymerization of ASC into highly cross-linked macromolecular assemblies called ASC specks or pyroptosomes [5]. ASC recruits pro-caspase-1, resulting in proximity-induced proteolytic cleavage of pro-caspase-1. Mature caspase-1 processes the pro-inflammatory cytokines interleukin-(IL)-1b and IL-18, as well as a range of other molecules. ASC specks are released by inflammasome-activated cells into the extracellular space and in body fluids where they continue to recruit and activate pro-caspase- 1 and to process the maturation of IL$b[6,7]$. Extracellular specks are taken up by neighboring cells and function to propagate the innate immune response [8]. ASC specks have been detected in tissues and sera of patients and mice with autoimmune and inflammatory diseases [6, 7], and serum levels of ASC are increased in MS patients [9]. The model of transcellular propagation of inflammasome activation via ASC specks suggests that extracellular ASC might be vulnerable to antibody-mediated therapies. We and others have observed that passive immunization with anti-ASC antibodies reduces pathology in several mouse models of excessive inflammasome activation [10-14]. In support of this idea is the finding that anti-Tau antibodies promote uptake of Tau aggregates into microglia and that these effects are epitope and aggregate size-dependent [15]. These studies therefore identify microglia as potential important effectors of therapeutic antibodies and demonstrate that anti-Tau antibodies are taken up by microglia and facilitate the clearance of extracellular Tau [15].

Emerging evidence has indicated important inflammasome-independent roles for ASC in controlling adaptive immune responses [16-19]. ASC regulates adaptive immunity by modulating Dock-2-dependent Rac activation and actin polymerization in dendritic cells and lymphocytes thereby regulating the motility of $T$ lymphocytes and $B$ lymphocytes and antigen uptake by professional antigen-presenting cells [17]. Thus, the vital role of ASC in regulating both adaptive and innate immune responses suggests that strategies to modulate ASC expression appear to be promising approaches for the treatment of CNS inflammatory diseases.

The NLRP3 and NLRC4 inflammasome have been previously associated with the pathology of MS [20, 21]. The NLRP3 inflammasome appears critical in the development of EAE, as demonstrated by the resistance of ${\mathrm{N} / r p 3^{-/-}}$mice to EAE $[22,23]$. The lack of the NLRP3 inflammasome in antigen-presenting cells dampens expression of chemokines and chemokine receptors on T helper cells and antigenpresenting cells, and prevents their migration to the CNS [23]. Alternatively, gene deletion of NLRP3 inflammasome components in mice subjected to EAE showed that progression of EAE is dependent on the inflammasome adaptor protein ASC and caspase-1, but not NLRP3 [19]. ASC ${ }^{-/}$mice were protected from EAE more than caspase- $1 \%$ mice, suggesting that an inflammasome-independent function of ASC contributes to EAE progression. The deficiency in ASC did not affect MOG-specific T cell proliferation or cytokine production in the periphery. However, ASC appeared to play a role in the peripheral survival of mature $\mathrm{CD} 4^{+} \mathrm{T}$ cells [19]. ASC ${ }^{--}$mice showed reduced numbers of MOG-specific $\mathrm{T}$ cells in the lymph nodes and in the CNS, resulting in protection from EAE. To explain these discrepancies, it has been suggested that EAE induced by weak activation of innate immunity requires the NLRP3 inflammasome, 
whereas strong activation of innate immunity makes the EAE pathological mechanism bypass the NLRP3 inflammasome and causing EAE to develop without the NLRP3 inflammasome [23].

Monoclonal antibodies (mAb) have been effectively used in MS therapy [24]. One of their main advantages is that, due of their high target specificity, they have minimal unwanted side effects. Natalizumab, which targets a4b1 integrin, and alemtuzumab, directed against the lymphocyte surface marker CD52, are among the most effective mAb currently in clinical use [24]. Recently, ocrelizumab, targeting the $\mathrm{B}$ cell antigen $\mathrm{CD} 20$, has been the first drug approved by the U.S. Food and Drug Administration (FDA) for PPMS [25-27]. Given the proven involvement of the inflammasome in MS and EAE pathobiology, we sought to pursue the testing of a mAb targeting ASC and ASC specks to improve clinical outcomes in EAE and MS.

In this study, we evaluated the therapeutic efficacy of a humanized monoclonal antibody against human ASC, IC100, in MOG-induced EAE. We show that IC100 significantly suppressed disease severity when compared to vehicle-treated controls. At a dose of $30 \mathrm{mg} / \mathrm{kg}, \mathrm{IC1} 100$ reduced the number of $\mathrm{CD} 4^{+}$and $\mathrm{CD}^{+} \mathrm{T}$ cells and $\mathrm{CD} 11 \mathrm{~b}^{+} \mathrm{MHCl}^{+}$activated myeloid cells in the spinal cord. In parallel, it reduced the number of total and activated microglial cells in the spinal cord. These data indicate that IC100 suppresses the adaptive and innate immune-inflammatory response that drives $E A E$, thereby identifying ASC as a promising target for MS therapy.

\section{Methods}

\section{Induction of EAE and development and treatment with IC-100}

Active EAE was induced in 2-months old C57BL/6 female mice with myelin oligodendrocyte glycoprotein 35-55 peptide ( $\mathrm{MOG}_{35-55}$, BioSynthesis) as previously described [28]. Briefly, mice received an intraperitoneal (i.p.) injection of pertussis toxin dissolved in PBS ( $350 \mathrm{ng} /$ mouse; day 0), followed by subcutaneous administration of $\mathrm{MOG}_{35-55}(300 \mathrm{ng} /$ mouse; day 1) emulsified in Complete Freund's Adjuvant, and a second i.p. injection of pertussis toxin (350 ng/mouse; day 2). IC100 (IgG4) was developed by humanization of a mouse monoclonal (IgG1) against human ASC (Abzena, Cambridge England). IC100 was cloned into a $\mathrm{CHO}$ cell manufacturing cell line (Selexis, Geneva, Switzerland). IC100 was purified from $\mathrm{CHO}$ cell supernatants using ProSepA high capacity column chromatography (Antibody Solutions, Santa Clara, CA). Mice were administered vehicle ( $0.9 \%$ saline) or IC100 at three different doses $(10,30$ and $45 \mathrm{mg} / \mathrm{kg}$ ) via i.p. injection every 4 days, starting at day 8 after induction of EAE. Clinical symptoms of EAE were assessed daily by a blind investigator on a scale of 0 to 6 as follows: 0 , no clinical signs; 1 , loss of tail tone; 2 , completely flaccid tail; 3 , complete hind limb paralysis; 4 , complete forelimb paralysis; 5 , moribund; 6 , dead. All experiments were performed according to protocols and guidelines approved by the Institutional Animal Care and Use Committee of the University of Miami.

\section{Cell isolation for flow cytometry}


Following transcardial perfusion with PBS, spinal cords were harvested and placed in cold Hanks' Balanced Salt Solution without $\mathrm{Mg}^{2+}$ and $\mathrm{Ca}^{2+}$ (HBSS w/o). Samples were then manually dissociated into single cell suspensions through a $70 \mathrm{~mm}$ strainer and washed in HBSS w/o. Spleen samples were spun at $1200 \mathrm{rpm}$ for $10 \mathrm{~min}$ at $4^{\circ} \mathrm{C}$, supernatants were removed, and red blood cells (RBCs) lysed in $2 \mathrm{ml}$ RBC lysis buffer (eBioscience) according the manufacturer's instructions. Spleen cells were then resuspended in PBS. Cells isolated from the spinal cord were resuspended in flow cytometry buffer (FCB, eBioscience), and incubated with Myelin Removal Beads II (Miltenyi). Myelin was depleted using the LS magnetic columns as described in the manufacturer's protocol (Miltenyi). Similar to the splenocytes, spinal cord cells were resuspended in PBS and stained as described below.

\section{Immunolabeling and flow cytometric analysis}

Cells were resuspended in $100 \mathrm{ml} \mathrm{FCB}$, blocked with $0.5 \mathrm{ml}$ TruStainFcX (anti-mouse CD16/32 FcR block, Biolegend) for $5 \mathrm{~min}$ at room temperature, and stained for $30 \mathrm{~min}$ at $4^{\circ} \mathrm{C}$ with: FITC-anti-CD45 (1:1000, Biolegend), PE-Cy7-anti-CD4 (1:200, eBioscience), PerCP-Cy5.5-anti-CD8 (1:200, Biolegend), PE-anti-B220 (1:200, Biolegend), APC-anti-MHCII (1:200, eBioscience), and APC-eFluor780-anti-CD11b (1:200, eBioscience) (Supplemental Table 1). Cells were then fixed in 1\% PFA for $1 \mathrm{~h}$. Finally, cells were resuspended in $500 \mathrm{ml} \mathrm{FCB}$, labelled with DAPI to exclude cell debris and analyzed with a CytoFLEX $\mathrm{S}$ flow cytometer (Beckman-Coulter) equipped with CytExpert software (Beckman-Coulter).

\section{Quantification of IC100 in Tissues.}

IC100 was quantified in brain, spinal cord, liver and spleen at 35 days post-induction (dpi) of EAE using a proprietary assay developed by InflamaCORE, LLC using Meso Scale Technology. Protein lysates were obtained as described in [29]. The assay was read using the QuickPlex SQ 120 instrument (Meso Scale Diagnostics, Maryland).

\section{Statistical Analyses}

EAE curves were analyzed point-by-point by 2-way repeated measures ANOVA, followed by Tukey test for multiple comparisons. The EAE curves as a whole were also analyzed by non-parametric Mann-Whitney test comparing treated groups to the control group, individually. All other assessments were analyzed by Student's $t$ test, if the data were normally distributed, or by non-parametric Mann-Whitney test if the data were not normally distributed. Normality was determined by the Pearson \& D'Agostino test. Data were expressed as mean \pm SEM, and $p$ values equal or less than 0.05 were considered statistically significant. Statistical analyses were carried out with GraphPad Prism software.

\section{Results}

Treatment with IC100 ameliorates the functional outcome in EAE 
In order to assess the therapeutic potential of IC100, we induced EAE with $\mathrm{MOG}_{35-55}$ peptide [30] in two months-old female C57BL/ 6 mice and administered IC100 or vehicle (saline) alone beginning 8 days post-induction (dpi) of the disease. The administration was repeated every 4 days until sacrifice ( $35 \mathrm{dpi}$ ). Three doses were tested, 10,30 and $45 \mathrm{mg} / \mathrm{kg}$. IC100 significantly improved functional recovery when used at the doses of 30 and $45 \mathrm{mg} / \mathrm{kg}$, with a robust reduction of the clinical disease scores throughout the duration of the experiment (Fig. 1A). The average peak clinical score was significantly reduced at both the 30 and $45 \mathrm{mg} / \mathrm{kg}$ doses (Fig. 1D, H), and the overall severity of EAE measured with the cumulative disease index (CDI) was reduced by half with the $30 \mathrm{mg} / \mathrm{kg}$ dose (Fig. 1C). Because the beneficial effect reached a plateau at $30 \mathrm{mg} / \mathrm{kg}$, we cannot exclude we may be able to lower the dose further or reduce the frequency of administration and obtain comparable or even enhanced benefits as the $30 \mathrm{mg} / \mathrm{kg}$ dose used here, while minimizing the chances of developing neutralizing antibodies against IC100 that may abrogate its effect.

\section{Treatment with IC100 reduces the infiltration of peripheral immune cells into the spinal cord following EAE}

Initiation, persistence and severity of the clinical symptoms in EAE are directly correlated with the infiltration of immune cells into the spinal cord. To evaluate whether IC100 altered this process, we analyzed by flow cytometry the profile of the immune cell populations isolated from the spinal cord at 35 dpi. Treatment with the effective dose of $30 \mathrm{mg} / \mathrm{kg}$ significantly reduced the total number of encephalitogenic $\mathrm{CD} 4^{+} \mathrm{T}$ cells as well as $\mathrm{CD} 8^{+} \mathrm{T}$ cells (Fig. $2 \mathrm{~A}, \mathrm{~B}$ ), the immune cell populations most crucial in driving EAE pathology. Surprisingly, the $45 \mathrm{mg} / \mathrm{kg}$ dose did not produce a reduction in spinal cord immune cell infiltration, despite reducing the overall severity of EAE. However, it is possible that at the high dose animals developed neutralizing antibodies against IC100 that diminished potency of the drug. On the other hand, it is important to note that immune cell infiltration was measured at $35 \mathrm{dpi}$, point beyond the acute phase of $E A E$, and it is possible that a second wave of immune activation contributes to the overall pathology. Indeed, this idea correlates with the mild elevation of the average clinical scores observed after $30 \mathrm{dpi}$ in the $45 \mathrm{mg} / \mathrm{kg}$ treated group.

Except for the T cell population, the numbers of all other measured immune cell populations did not change, although a clear downward trend was observed particularly for CD11 $\mathrm{b}^{+}$myeloid cells (macrophages and neutrophils combined) (Fig. 2A, B). No differences in cell numbers were observed with any of the IC100 doses in the spleen, suggesting treatment did not interfere with the ability of mice to mount an adequate immune response to the EAE challenge (Fig. 3). The gating strategy for the flow cytometry experiments is reported in Supplementary Figure 1.

\section{Treatment with IC100 reduces the number and activation state of microglia following EAE}

Microglia participate in the immune-inflammatory response associated with CNS disease. Their activation is characterized by increased proliferation and upregulation of $\mathrm{MHCll}$ surface expression. To assess whether IC100 increased microglial cell activation, we quantified the number of total microglia, 
identified as the $\mathrm{CD} 45^{\text {low }} \mathrm{CD} 11 \mathrm{~b}^{+}$population (Fig. $4 \mathrm{~B}$, green circles), and $\mathrm{MHCII}$ activated microglia (Fig. $4 \mathrm{~B}$, right panels) in the same spinal cord samples by flow cytometry. Total microglia were highly reduced by $\mathrm{IC} 100$ at $30 \mathrm{mg} / \mathrm{kg}$, and so were the $\mathrm{MHCl}^{+}$activated microglia (Fig. 4A). This finding was confirmed by Iba1 immunostaining of spinal cord tissue sections where vehicle treated mice displayed a clear increase in microglia numbers throughout the white and gray matter compared to the $30 \mathrm{mg} / \mathrm{kg}$ IC100 treated group (Fig. 4C). In the vehicle treated group microglia also appeared more ramified and hypertrophic. Together, these data indicate that IC100 is effective in suppressing microglia activation and microglia-mediated neuroinflammation.

\section{IC100 penetrates the brain and spinal cord}

An important parameter in designing drugs to treat MS, particularly if they are meant to target neuroinflammation, is to determine whether they penetrate and accumulate in the CNS at therapeutic concentrations. This is especially important in the treatment of the progressive MS forms, where the blood-brain barrier appears relatively intact [31]. Thus, we quantified the levels of IC100 in brain, spinal cord, liver and spleen. IC100 penetrated the tissues analyzed at all three dosages (Fig. 5). The levels of IC100 in the spinal cord and brain were maximal at the 30 and $45 \mathrm{mg} / \mathrm{kg}$ doses, consistent with their therapeutic effect on reducing EAE severity (Fig. 1).

\section{Discussion}

In this study we investigated the functional and immunological effects of IC100, a monoclonal antibody against the inflammasome protein ASC, in the EAE animal model of MS. Here we show that ASC plays a critical role in the development of EAE, and that, by blocking ASC, the adaptive and innate immune response that sustain EAE are significantly reduced. Indeed, IC100 decreased the number of $\mathrm{CD} 4^{+}, \mathrm{CD} 8^{+} \mathrm{T}$ and $\mathrm{CD} 11 \mathrm{~b}^{+} \mathrm{MHCII}{ }^{+}$cells trafficking into the CNS, while reducing the number and activation state of CNS resident microglia. This is the first report to show that targeting ASC with a monoclonal antibody in MOGinduced EAE significantly alters innate and adaptive immune responses leading to improved clinical outcomes. Importantly, this work constitutes a novel approach to identify new drugs targeting the inflammasome that could be useful for the treatment of MS.

Our previous studies have shown that ASC is a reliable disease biomarker found in serum of patients with MS [9], traumatic brain injury [32,33] and depression [25], as well as in extracellular vesicles isolated from individuals with stroke [11]. It is possible that IC100 works extracellularly to prevent ASC oligomerization into highly cross-linked macromolecular assemblies. On the other hand, ASC or ASC-IC100 complexes may be taken up by cells and internalized to prevent recruitment of ASC into the inflammasome complex. Previous studies in our laboratory have shown that anti-ASC is taken up by spinal cord neurons after trauma [10], and by the human monocytic leukemia cell line (THP-1 cells, unpublished data). It has been shown that monoclonal antibodies enter cells by receptor- mediated endocytosis through binding of the Fc domain to Fc-gamma-receptors (FcgRs) expressed on many immune cells, including monocytes, macrophages, microglia, myeloid progenitor cells and dendritic cells $[34,35]$. However, it is generally 
accepted that low affinity FcgRs differ from other receptors in that their activation requires cross-linking of polyvalent immune complexes, such as when multiple antibodies are bound to a protein aggregate [36, 37]. Multiple studies have suggested a role for microglia-mediated clearance of aggregated proteins as asynuclein [38], Ab aggregates $(31,34)$ and Tau [15]. The later study also observed a clear size dependence of the microglial clearance pathway. Since ASC is secreted and oligomerizes into ASC specks, it is possible that IC100 may identify ASC assemblies and then are taken up by immune cells by receptor-mediated endocytosis. Therefore, establishing the precise specific mechanism of IC100 activity will help to design and optimize efficacy in vivo.

IL-1b/IL-18 secreted after inflammasome activation induce neutrophils and macrophages to engulf pathogens, promote inflammatory cell recruitment, and further amplify inflammatory responses $[39,40]$. Apart from its impact on innate immunity, inflammasome activation also initiates adaptive immunity through the modulation of T helper subsets, skewing T cell differentiation in favor of Th1 and Th17 phenotypes [41, 42]. IL-1b also activates IL-1R signaling, which has a substantial influence on Th17 cell differentiation and related immune responses [43]. Several studies with $\mathrm{ASC}^{-/-}$mice have suggested inflammasome-independent roles of ASC in the adaptive immune response. A novel microbial pathogeninduced mechanism for ASC in activating chemokine expression through MAPK activation, independent of the conventional caspase-1 inflammasome, has been reported [44]. Two studies of antigen-induced murine arthritis show dependence on ASC, but caspase-1, NLRP3, and NLRC4 independence [17]. A requirement for ASC, but not NLRP3 or caspase-1, was also demonstrated for antigen-specific humoral immunity after vaccination with MF59-adjuvented influenza [16]. In addition, $\mathrm{ASC}^{-/}$- mice have reduced numbers of MOG-specific T cells in the lymph nodes and CNS, resulting in protection from EAE [19]. Lastly, genetic evidence shows an unexpected role of ASC in regulating motility of T lymphocytes and Blymphocytes and antigen uptake by professional antigen-presenting cells independently of inflammasomes [17]. In this study, ASC was shown to regulate the mRNA stability and expression of Dock2, a guanine nucleotide-exchange factor that mediates Rac-dependent signaling in immune cells [17].

The blood-brain barrier (BBB) presents a great impediment for brain drug delivery in that it is questioned whether sufficient intralesional drug concentrations can be reached after systemic administration. This problem is especially evident for biologicals such as monoclonal antibodies (mAbs) that are relatively large molecules. To overcome the presumed drug delivery obstacle in the brain, many treatment strategies for CNS diseases are directed at disrupting, passing, or bypassing the effective BBB $[46,47]$. Disruption has been tried chemically, by drugs that influence passive diffusion (e.g., bradykinin, mannitol, regadenoson, and borneol) or active transport mechanisms (e.g., elacridar), or by radiotherapy, ultrasound, or microwaves. Moreover, via viral vectors, nanoparticles, liposomes, exosomes, and transporter or receptor ligands have been tried to bypass the BBB $[46,47]$.

However, the BBB is disrupted under various pathological conditions of diseases such as stroke, diabetes, seizures, hypertensive encephalopathy, acquired immunodeficiency syndrome, traumatic brain injuries, multiple sclerosis, Parkinson's disease (PD) and Alzheimer disease (AD) [47]. It appears that in some 
pathological conditions, remodeling of the protein complex in interendothelial junctions is an important reason for the BBB breakdown [47]. Therefore, in CNS disease conditions associated with a leaky BBB, mAbs have been widely explored because of their specificity and high affinity for critical disease targets. Additionally, other important areas that deserve further investigation are the influence of aging [47] and major depressive disorder [48] on BBB dysfunctions and to discover whether mAbs may be used as possible approaches to deliver drugs into the brain to treat these conditions.

\section{Conclusions}

Our data reveal that administration of IC100 $(30 \mathrm{mg} / \mathrm{kg})$ in MOG-induced EAE resulted in significant decrease in the number of $\mathrm{CD} 4^{+}, \mathrm{CD} 8^{+}$and $\mathrm{CD} 11 \mathrm{~b}^{+} \mathrm{MHCll}^{+}$cells in the spinal cord resulting in protection from EAE. In addition, this dose of IC100 also decreased the numbers of total and activated microglia in the spinal cord and resulted in improved clinical scores. These findings are consistent with our previous studies demonstrating that antibody-mediated neutralization of ASC results in significant functional improvements in spinal cord [10] and brain trauma [45] injury models. Nevertheless, further studies are needed to uncover specific mechanisms of IC100 antibody activity and whether IC100 influences inflammasome-dependent and/or inflammasome-independent signaling cascades modulating adaptive and innate immune cell functions, and/or the trafficking of lymphocytes and myeloid cells into the CNS.

Decades of pharmacological research in EAE and MS have led to the development of mAbs against a variety of targets which have revolutionized MS treatment with an improvement in effectiveness. In the present study we have discovered that IC100, which targets the inflammasome adaptor protein ASC, exhibits therapeutic properties in EAE and suppresses immune cell responses underlying tissue destruction. These findings provide a basis for the further development of anti-ASC drugs for the treatment not only of MS, but also for other neurodegenerative and traumatic CNS pathologies.

\section{Abbreviations}

ASC: apoptosis-associated speck-like protein containing a caspase-recruitment domain; MS: multiple sclerosis; EAE: experimental autoimmune encephalomyelitis; MOG: myelin oligodendrocyte glycoprotein; CNS: central nervous system; RRMS: relapsing-remitting; SPMS: secondary-progressive; IL: interleukin; DOCK-2: Dedicator of cytokinesis 2; NLRP: NOD-like receptor protein; NLRC4: NLR Family CARD Domain Containing 4; mAb: monoclonal antibodies; FDA: Food and Drug Administration; CD: Cluster of Differentiation; IgG: immunoglobulin; HBSS w/o: Hanks' Balanced Salt Solution without Mg2+ and Ca2+; RBC: red blood cells; FCB: flow cytometry buffer; SEM: Standard error of the mean; dpi: days postinduction CDI: cumulative disease index; Th: T helper

\section{Declarations}

\section{Authors' contributions}


HLD, MP, PI, JPdRV and RB conducted experiments and analyzed data. RWK, RB, JPdRV, WDD and HMB conceived the study. All authors were involved in the writing and reviewing of the manuscript.

\section{Funding}

This work was supported by the Coulter Foundation (RWK, JPdRV) and the NIH NINDS grant 1R01NS094522-01 (RB) and R01NS113969-01 (RWK, JPdRV).

\section{Availability of data and materials}

The data that support the findings are not publicly available. Data are available from the authors upon request.

\section{Ethics approval and consent to participate}

The experiments were approved by the Institutional Animal Care and Use Committee at the University of Miami (IACUC protocol number: 17-086)

\section{Competing Interests}

JPdRV, RWK, WDD and HMB are co-founders and managing members of InflamaCORE, LLC and have licensed patents on inflammasome proteins as biomarkers of injury and disease as well as on targeting inflammasome proteins for therapeutic purposes. JPdRV, RWK, WDD and HMB are Scientific Advisory Board Members of ZyVersa Therapeutics, Inc. RB has licensed a patent on inflammasome proteins as a therapy for MS. All other authors declare that there are no conflicts of interest.

\section{Consent for Publication:}

Not applicable

\section{Acknowledgments:}

Not applicable

\section{References}

1. Nylander A, Hafler DA: Multiple sclerosis. J Clin Invest 2012, 122:1180-1188.

2. Compston A, Coles A: Multiple sclerosis. Lancet 2008, 372:1502-1517.

3. Dobson R, Giovannoni G: Multiple sclerosis - a review. Eur J Neurol 2019, 26:27-40.

4. Constantinescu CS, Farooqi N, O'Brien K, Gran B: Experimental autoimmune encephalomyelitis (EAE) as a model for multiple sclerosis (MS). Br J Pharmacol 2011, 164:1079-1106. 
5. de Alba E: Structure, interactions and self-assembly of ASC-dependent inflammasomes. Arch Biochem Biophys 2019.

6. Franklin BS, Bossaller L, De Nardo D, Ratter JM, Stutz A, Engels G, Brenker C, Nordhoff M, Mirandola SR, Al-Amoudi A, et al: The adaptor ASC has extracellular and 'prionoid' activities that propagate inflammation. Nat Immunol 2014, 15:727-737.

7. Baroja-Mazo A, Martin-Sanchez F, Gomez Al, Martinez CM, Amores-Iniesta J, Compan V, BarberaCremades $\mathrm{M}$, Yague J, Ruiz-Ortiz E, Anton J, et al: The NLRP3 inflammasome is released as a particulate danger signal that amplifies the inflammatory response. Nat Immuno/ 2014, 15:738-748.

8. Franklin BS, Latz E, Schmidt FI: The intra- and extracellular functions of ASC specks. Immunol Rev 2018, 281:74-87.

9. Keane RW, Dietrich WD, de Rivero Vaccari JP: Inflammasome Proteins As Biomarkers of Multiple Sclerosis. Front Neurol 2018, 9:135.

10. de Rivero Vaccari JP, Lotocki G, Marcillo AE, Dietrich WD, Keane RW: A molecular platform in neurons regulates inflammation after spinal cord injury. $J$ Neurosci 2008, 28:3404-3414.

11. Kerr N, Garcia-Contreras M, Abbassi S, Mejias NH, Desousa BR, Ricordi C, Dietrich WD, Keane RW, de Rivero Vaccari JP: Inflammasome Proteins in Serum and Serum-Derived Extracellular Vesicles as Biomarkers of Stroke. Front Mol Neurosci 2018, 11:309.

12. Kerr NA, de Rivero Vaccari JP, Umland O, Bullock MR, Conner GE, Dietrich WD, Keane RW: Human Lung Cell Pyroptosis Following Traumatic Brain Injury. Cells 2019, 8.

13. Lee SW, de Rivero Vaccari JP, Truettner JS, Dietrich WD, Keane RW: The role of microglial inflammasome activation in pyroptotic cell death following penetrating traumatic brain injury. $J$ Neuroinflammation 2019, 16:27.

14. Lee SW, Gajavelli S, Spurlock MS, Andreoni C, de Rivero Vaccari JP, Bullock MR, Keane RW, Dietrich WD: Microglial Inflammasome Activation in Penetrating Ballistic-Like Brain Injury. J Neurotrauma 2018, 35:1681-1693.

15. Funk KE, Mirbaha H, Jiang H, Holtzman DM, Diamond MI: Distinct Therapeutic Mechanisms of Tau Antibodies: Promoting Microglial Clearance Versus Blocking Neuronal Uptake. J Biol Chem 2015, 290:21652-21662.

16. Ellebedy AH, Lupfer C, Ghoneim HE, DeBeauchamp J, Kanneganti TD, Webby RJ: Inflammasomeindependent role of the apoptosis-associated speck-like protein containing CARD (ASC) in the adjuvant effect of MF59. Proc Natl Acad Sci U S A 2011, 108:2927-2932.

17. Ippagunta SK, Brand DD, Luo J, Boyd KL, Calabrese C, Stienstra R, Van de Veerdonk FL, Netea MG, Joosten LA, Lamkanfi M, Kanneganti TD: Inflammasome-independent role of apoptosis-associated speck-like protein containing a CARD (ASC) in T cell priming is critical for collagen-induced arthritis. J Biol Chem 2010, 285:12454-12462.

18. Kolly L, Karababa M, Joosten LA, Narayan S, Salvi R, Petrilli V, Tschopp J, van den Berg WB, So AK, Busso N: Inflammatory role of ASC in antigen-induced arthritis is independent of caspase-1, NALP-3, and IPAF. J Immunol 2009, 183:4003-4012. 
19. Shaw PJ, Lukens JR, Burns S, Chi H, McGargill MA, Kanneganti TD: Cutting edge: critical role for PYCARD/ASC in the development of experimental autoimmune encephalomyelitis. J Immuno/ 2010, 184:4610-4614.

20. Soares JL, Oliveira EM, Pontillo A: Variants in NLRP3 and NLRC4 inflammasome associate with susceptibility and severity of multiple sclerosis. Mult Scler Relat Disord 2019, 29:26-34.

21. Khan N, Kuo A, Brockman DA, Cooper MA, Smith MT: Pharmacological inhibition of the NLRP3 inflammasome as a potential target for multiple sclerosis induced central neuropathic pain. Inflammopharmacology 2018, 26:77-86.

22. Gris D, Ye Z, locca HA, Wen H, Craven RR, Gris P, Huang M, Schneider M, Miller SD, Ting JP: NLRP3 plays a critical role in the development of experimental autoimmune encephalomyelitis by mediating Th1 and Th17 responses. J Immunol 2010, 185:974-981.

23. Inoue M, Williams KL, Gunn MD, Shinohara ML: NLRP3 inflammasome induces chemotactic immune cell migration to the CNS in experimental autoimmune encephalomyelitis. Proc Natl Acad Sci U S A 2012, 109:10480-10485.

24. Voge NV, Alvarez E: Monoclonal Antibodies in Multiple Sclerosis: Present and Future. Biomedicines $2019,7$.

25. Syed YY: Ocrelizumab: A Review in Multiple Sclerosis. CNS Drugs 2018, 32:883-890.

26. Chin P, Chan AC: Ocrelizumab: A New Therapeutic Paradigm for Multiple SclerosisPublished as part of the Biochemistry series "Biochemistry to Bedside". Biochemistry 2018, 57:474-476.

27. Mulero $\mathrm{P}$, Midaglia L, Montalban X: Ocrelizumab: a new milestone in multiple sclerosis therapy. Ther Adv Neurol Disord 2018, 11:1756286418773025.

28. Gao H, Danzi MC, Choi CS, Taherian M, Dalby-Hansen C, Ellman DG, Madsen PM, Bixby JL, Lemmon VP, Lambertsen KL, Brambilla R: Opposing Functions of Microglial and Macrophagic TNFR2 in the Pathogenesis of Experimental Autoimmune Encephalomyelitis. Cell Rep 2017, 18:198-212.

29. Mejias NH, Martinez CC, Stephens ME, de Rivero Vaccari JP: Contribution of the inflammasome to inflammaging. J Inflamm (Lond) 2018, 15:23.

30. Brambilla R, Morton PD, Ashbaugh JJ, Karmally S, Lambertsen KL, Bethea JR: Astrocytes play a key role in EAE pathophysiology by orchestrating in the CNS the inflammatory response of resident and peripheral immune cells and by suppressing remyelination. Glia 2014, 62:452-467.

31. Lassmann $\mathrm{H}$, van Horssen J, Mahad D: Progressive multiple sclerosis: pathology and pathogenesis. Nat Rev Neurol 2012, 8:647-656.

32. Kerr N, Lee SW, Perez-Barcena J, Crespi C, Ibanez J, Bullock MR, Dietrich WD, Keane RW, de Rivero Vaccari JP: Inflammasome proteins as biomarkers of traumatic brain injury. PLoS One 2018, 13:e0210128.

33. Adamczak S, Dale G, de Rivero Vaccari JP, Bullock MR, Dietrich WD, Keane RW: Inflammasome proteins in cerebrospinal fluid of brain-injured patients as biomarkers of functional outcome: clinical article. J Neurosurg 2012, 117:1119-1125. 
34. Gessner JE, Heiken H, Tamm A, Schmidt RE: The IgG Fc receptor family. Ann Hemato/ 1998, 76:231248.

35. Ryman JT, Meibohm B: Pharmacokinetics of Monoclonal Antibodies. CPT Pharmacometrics Syst Pharmacol 2017, 6:576-588.

36. Garcia-Garcia E, Rosales C: Signal transduction during Fc receptor-mediated phagocytosis. J Leukoc Biol 2002, 72:1092-1108.

37. Metzger H, Kinet JP: How antibodies work: focus on Fc receptors. FASEB J 1988, 2:3-11.

38. Bae EJ, Lee HJ, Rockenstein E, Ho DH, Park EB, Yang NY, Desplats P, Masliah E, Lee SJ: Antibodyaided clearance of extracellular alpha-synuclein prevents cell-to-cell aggregate transmission. $J$ Neurosci 2012, 32:13454-13469.

39. He Y, Hara H, Nunez G: Mechanism and Regulation of NLRP3 Inflammasome Activation. Trends Biochem Sci 2016, 41:1012-1021.

40. van de Veerdonk FL, Netea MG, Dinarello CA, Joosten LA: Inflammasome activation and IL-1beta and IL-18 processing during infection. Trends Immunol 2011, 32:110-116.

41. Evavold CL, Kagan JC: How Inflammasomes Inform Adaptive Immunity. J Mol Biol 2018, 430:217237.

42. Mills KH, Dungan LS, Jones SA, Harris J: The role of inflammasome-derived IL-1 in driving IL-17 responses. J Leukoc Biol 2013, 93:489-497.

43. Chung Y, Chang SH, Martinez GJ, Yang XO, Nurieva R, Kang HS, Ma L, Watowich SS, Jetten AM, Tian Q, Dong C: Critical regulation of early Th17 cell differentiation by interleukin-1 signaling. Immunity 2009, 30:576-587.

44. Taxman DJ, Holley-Guthrie EA, Huang MT, Moore CB, Bergstralh DT, Allen IC, Lei Y, Gris D, Ting JP: The NLR adaptor ASC/PYCARD regulates DUSP10, mitogen-activated protein kinase (MAPK), and chemokine induction independent of the inflammasome. J Biol Chem 2011, 286:19605-19616.

45. de Rivero Vaccari JP, Lotocki G, Alonso OF, Bramlett HM, Dietrich WD, Keane RW: Therapeutic neutralization of the NLRP1 inflammasome reduces the innate immune response and improves histopathology after traumatic brain injury. J Cereb Blood Flow Metab 2009, 29:1251-1261.

46. Veldhuijzen van Zanten SEM, De Witt Hamer PC, van Dongen GAMS: Brain access of monoclonal antibodies as imaged and quantified by ${ }^{89} \mathrm{Zr}$-antibody PET: Perspectives for treatment of brain diseases. J. Nucl Med 2019, 60:615-616.

47. Dong X: Current strategies for brain drug delivery. Theranostics 2018, 8:1481-1493.

48. Cheung Y, Desse S, Martinez A, Worthen RJ, Jope RS, Beurel E: TNFa disrupts blood brain barrier integrity to maintain prolonged depressive-like behavior in mice. Brain Behav Immun. 2018, 69:556567.

\section{Supplementary Table 1}

Supplementary Table I. Antibodies for flow cytometry. 


\begin{tabular}{|l|l|l|l|l|}
\hline Antigen & Color & Dilution & Provider & Catalog \# \\
\hline CD45 & FITC & $1: 1000$ & eBioscience & $11-0451-82$ \\
\hline CD4 & PE/Cy7 & $1: 200$ & eBioscience & $25-0042-81$ \\
\hline CD8 & Percp-Cy 5.5 & $1: 200$ & Biolegend & 100734 \\
\hline B220 & PE & $1: 200$ & Biolegend & 103208 \\
\hline CD11b & APC eFluor780 & $1: 200$ & eBioscience & $47-0112-82$ \\
\hline MHCII & APC & $1: 200$ & eBioscience & $17-5321-81$ \\
\hline
\end{tabular}

Figures
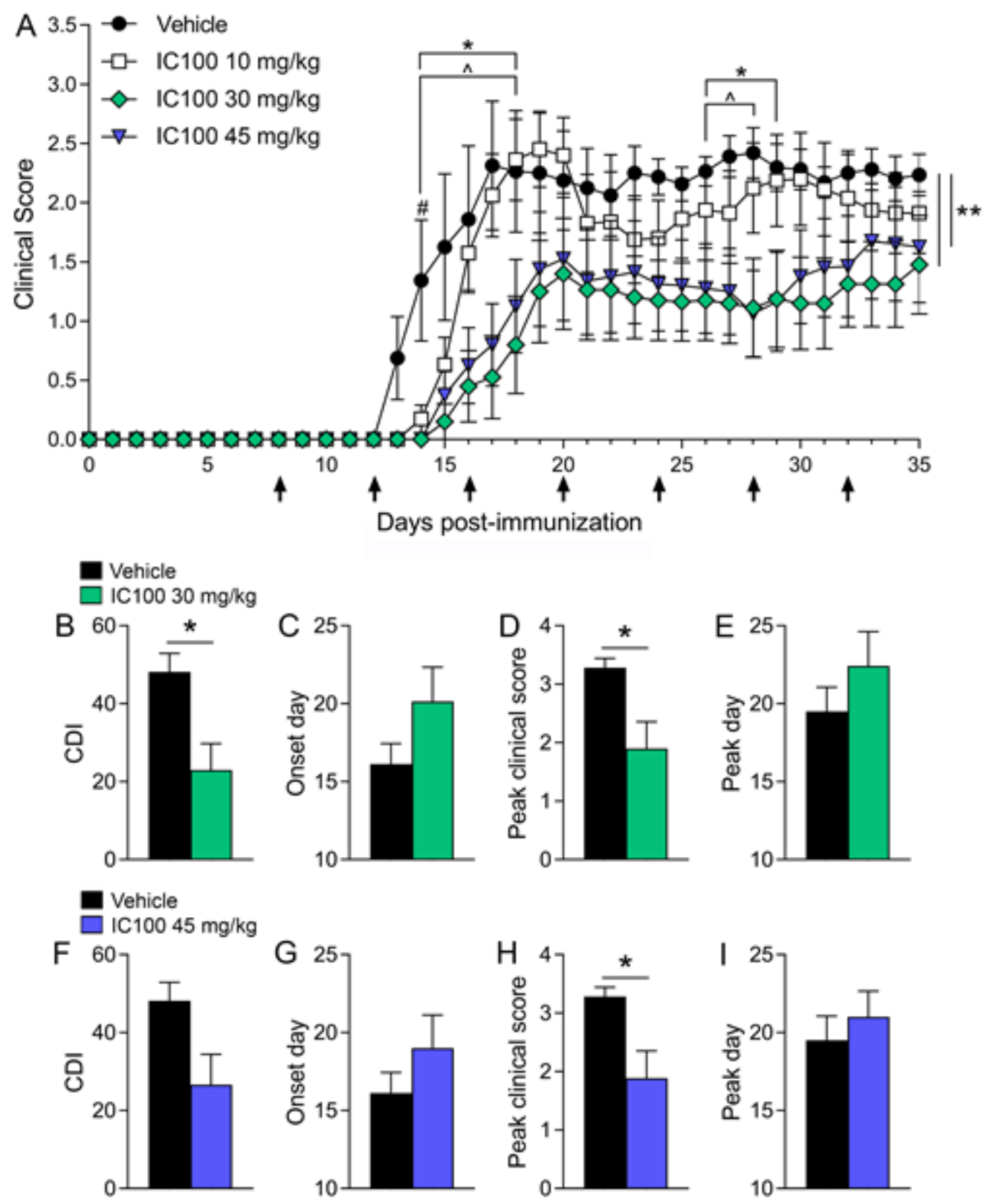

Figure 1 
IC100 treatment improves the functional outcome in EAE. (A) Clinical course of MOG35-55-induced EAE in C57BL/ 6 mice treated with vehicle or increasing doses of IC100 (10, 30 and $45 \mathrm{mg} / \mathrm{kg}$ i.p. every 4 days) with administration starting at day 8 . Results are expressed as daily mean clinical score \pm SEM of 9-10 mice/group. Statistical analysis by 2-way repeated measures ANOVA with Tukey's multiple comparisons test: \#Significantly different time point, Vehicle vs IC100 $10 \mathrm{mg} / \mathrm{kg}: \mathrm{p}$ value $14 \mathrm{dpi}=0.0313$.

*Significantly different time points, Vehicle vs IC100 $30 \mathrm{mg} / \mathrm{kg}$ : $\mathrm{p}$ values $14-18 \mathrm{dpi}=0.0089,0.0031$, $0.0053,0.0002,0.0034 ; \mathrm{p}$ values $27-30 \mathrm{dpi}=0.0191,0.0116,0.046,0.04{ }^{\wedge}{ }^{\wedge}$ Significantly different time points, Vehicle vs IC100 $45 \mathrm{mg} / \mathrm{kg}$ : p values 14-18 dpi $=0.0089,0.0179,0.02,0.0023,0.0377 ; \mathrm{p}$ values 27$29 \mathrm{dpi}=0.0377,0.0079,0.0392 .{ }^{* *}$ Curve comparisons: Vehicle vs IC100 $30 \mathrm{mg} / \mathrm{kg}, \mathrm{p}=0.0025$; Vehicle vs IC100 $45 \mathrm{mg} / \mathrm{kg}, \mathrm{p}=0.004$, Mann-Whitney test. (B-I) Comparison of EAE parameters between Vehicle treated mice and each of the two effective doses: (B, F) Cumulative Disease Index (CDI), calculated as sum of daily scores from day of onset for each animal (measure of EAE severity), * $p \leq 0.05$, MannWhitney test; $(C, G)$ Onset day, considered as day a mouse showed EAE symptoms for two consecutive days; (D, H) Peak clinical score, considered as highest score reached by a mouse acutely after onset, ${ }^{*} \mathrm{p} \leq 0.05$, Student's $t$ test; $(E, I)$ Peak disease day, considered as day a mouse reached the highest disease score acutely after onset. 

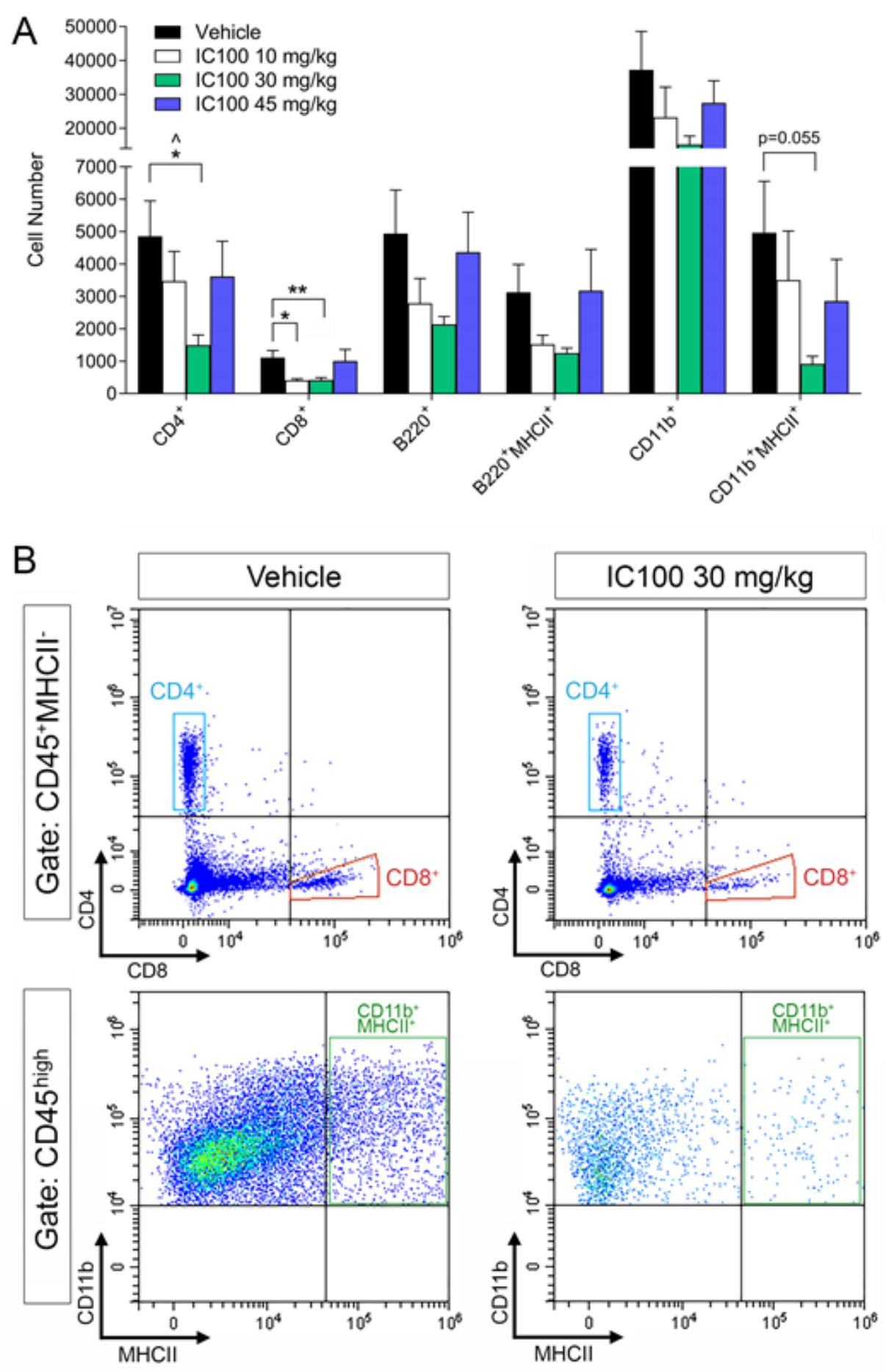

\section{Figure 2}

IC100 treatment reduces peripheral immune cell infiltration into the spinal cord following EAE. (A) Flow cytometric quantification of the leukocyte populations infiltrating into the spinal cord at $35 \mathrm{dpi}$ after EAE. The flow cytometry gating strategy is provided in Supplementary Figure 1. (B) Representative flow cytometry plots of CD4 and CD8 T cell populations from mice treated with vehicle and IC100 $(30 \mathrm{mg} / \mathrm{kg})$. In order to assure that no dendritic cells expressing CD4 or CD8 were included, we identified the CD4+ and 
CD8+ T cell populations after gating on the CD45+MHCll-population. Results are expressed as average \pm SEM of 5 mice/group, ${ }^{*} p<0.05$, ${ }^{*} p<0.001$, Mann-Whitney $t$ test; ${ }^{\wedge} p<0.05$, one-way ANOVA, Dunnet's multiple comparisons test.

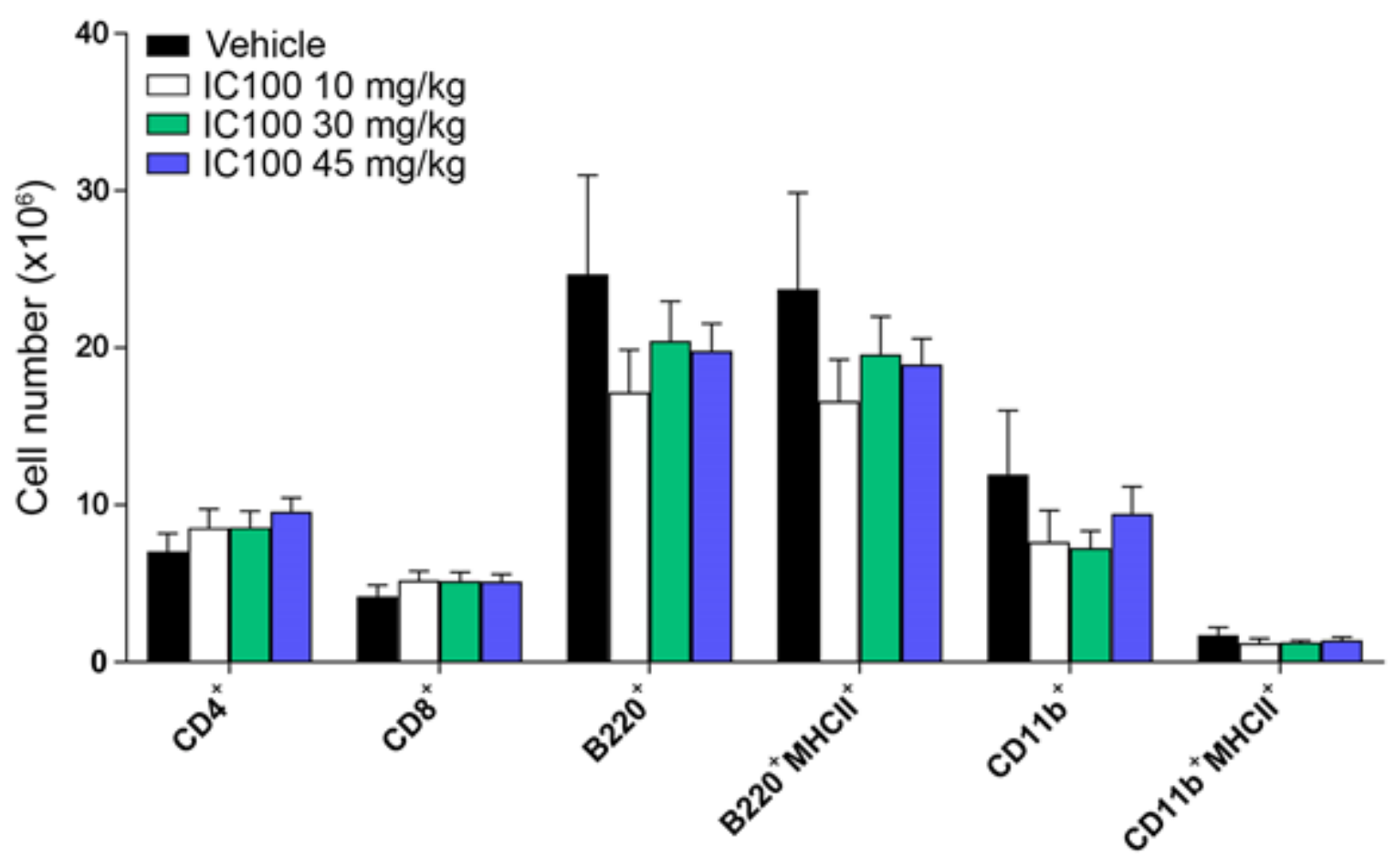

Figure 3

IC100 treatment does not affect splenic immune cell populations following EAE. Flow cytometric quantification of the leukocyte populations in the spleen at $35 \mathrm{dpi}$ EAE. Results are expressed as average \pm SEM of 5 mice/group. 

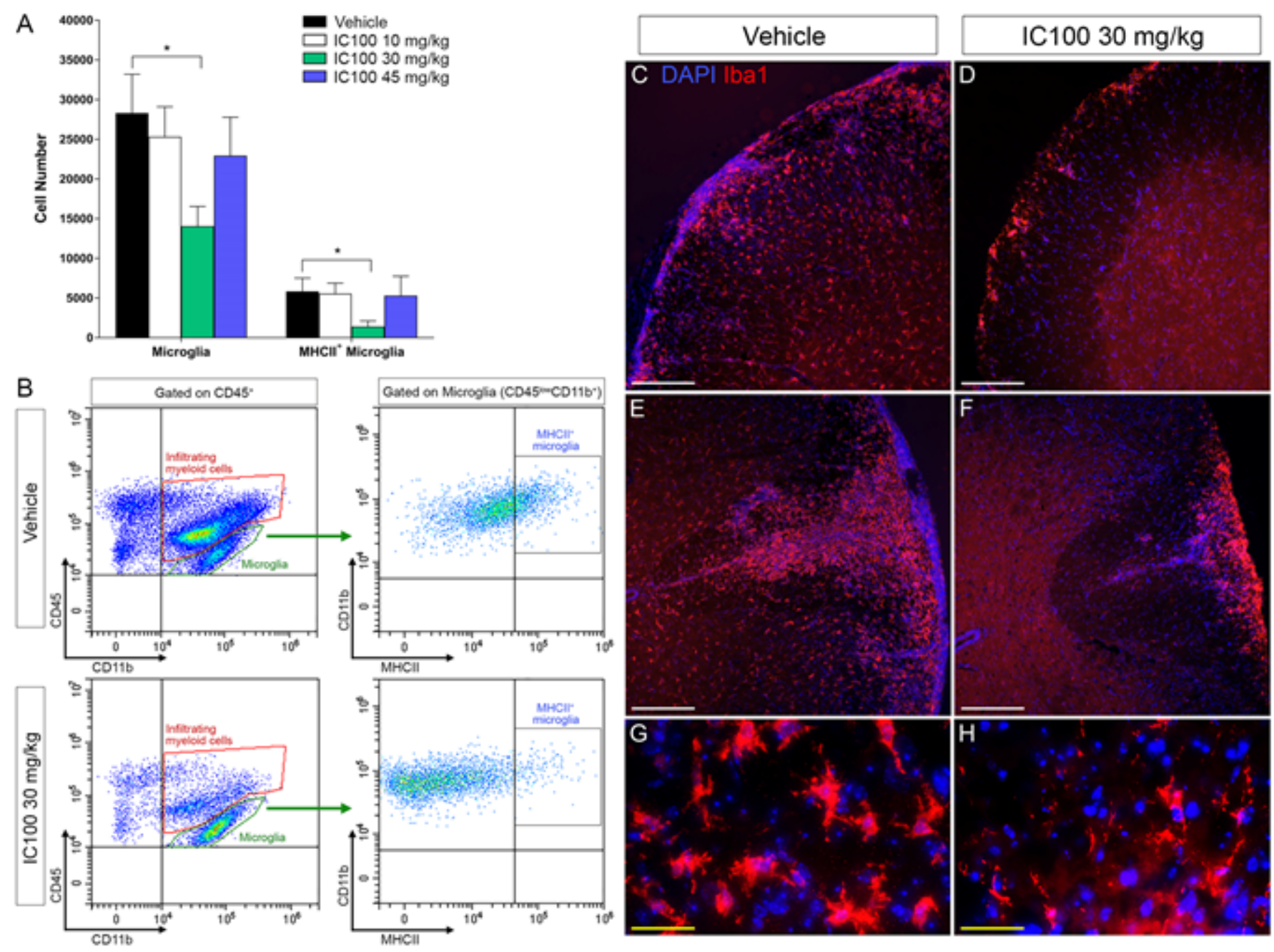

\section{Figure 4}

IC100 treatment reduces number and activation state of microglia following EAE. (A) Flow cytometric quantification of CD45lowCD11 b+ total microglia and CD45lowCD11b+MHCII+ activated microglia in the spinal cord at $35 \mathrm{dpi}$ after EAE. (B) Representative flow cytometry plots of CD45lowCD11b+total microglia (green circles) and CD45lowCD11b+MHCll+ activated microglia (right panels) from mice treated with vehicle and IC100 (30 mg/kg). Results are expressed as average \pm SEM of 5 mice/group, ${ }^{*} p<0.05$, Student's $t$ test; no statistically significant differences were observed by one-way ANOVA with Dunnet's multiple comparisons test (C) Iba1 immunostaining of microglia and infiltrating myeloid cells in the spinal cord of mice treated with vehicle and IC100 (30 mg/kg). White scale bar $=200 \square \mathrm{m}$; yellow scale bar $=50 \square \mathrm{m}$. 


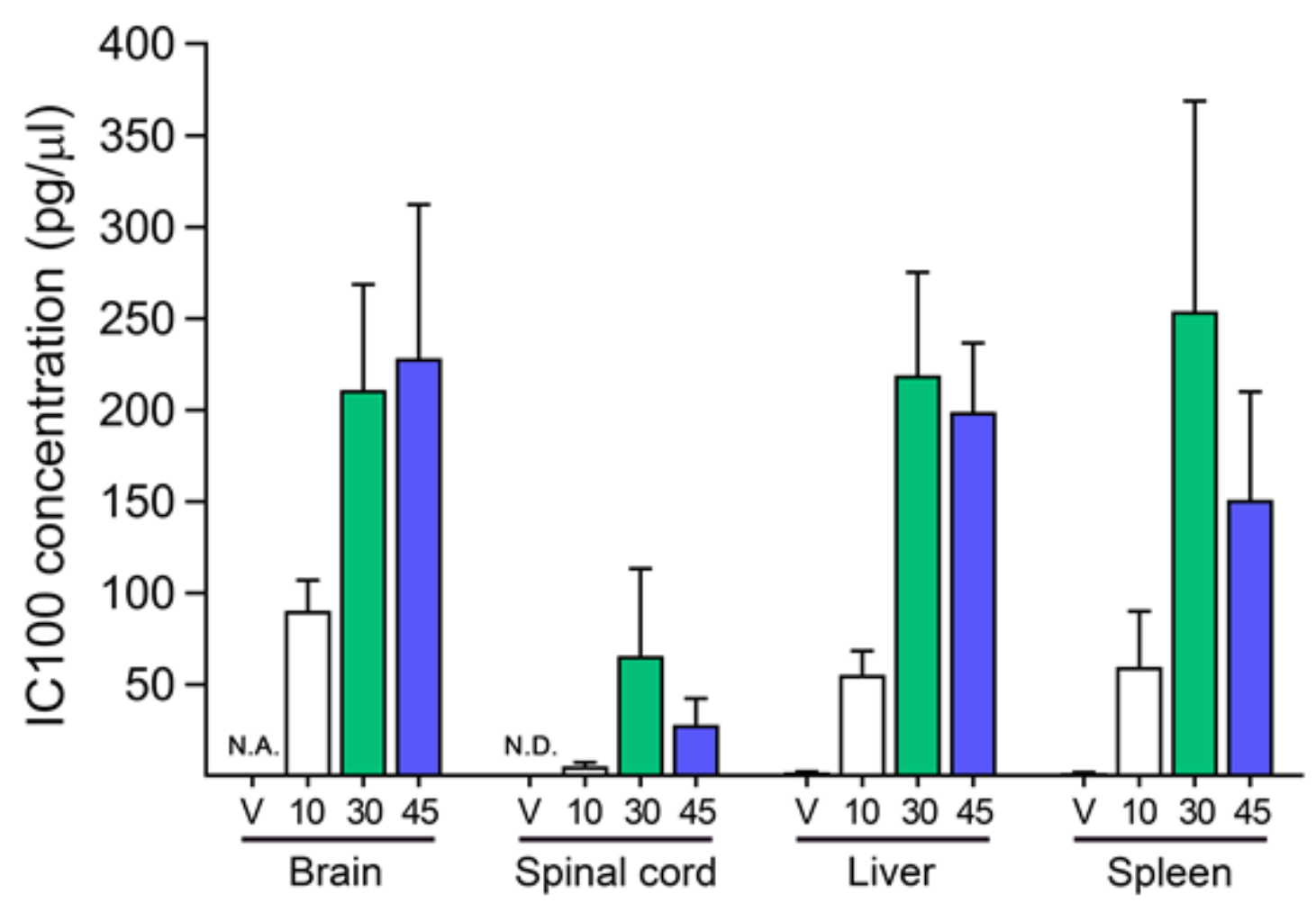

Figure 5

IC100 concentration in tissues. Concentrations of IC100 in pg/DI in the brain, spinal cord, liver and spleen of mice treated with IC100 at 10, 30 and $45 \mathrm{mg} / \mathrm{kg}$ or vehicle at $35 \mathrm{dpi}$ EAE. Results are expressed as mean \pm SEM of 5-10 mice/group. N.A. = not assessed; N.D. = not detected.

\section{Supplementary Files}

This is a list of supplementary files associated with this preprint. Click to download.

- SupplementaryFigure1.jpg 